\title{
Stability analysis of Automatic Voltage Regulator using Fractional Order Controller
}

\author{
K Muralidhar Goud ${ }^{a}$, C. Srisailam ${ }^{b}$, D. Sathish ${ }^{\text {, }}$, and D. Harshad \\ ${ }^{a}$ K Muralidhar Goud, Department of EEE, Vardhaman College of Engineering, Shamshabad, Hyderabad. \\ ${ }^{\mathrm{b} C}$ Srisailam, Department of EEE, ChaitanyaBharathi Institute of Technology (A), Hyderabad. \\ ${ }^{c}$ D. Sathish, Department of EEE, ChaitanyaBharathi Institute of Technology (A), Hyderabad. \\ ${ }^{\mathrm{d}}$ D. Harsha, Department of EEE, ChaitanyaBharathi Institute of Technology (A), Hyderabad.
}

Article History: Received: 11 January 2021; Accepted: 27 February 2021; Published online: 5 April 2021

Abstract: We aim to design a fractional order robust control system. It is an advanced model of classic PID controller whose order will be non-integer.PID controller that we generally use has many advantages and disadvantages with respect to the disadvantages like, it doesn't give accurate values of constants, exact values of the time domain parameters as well as frequency domain parameters of the control system and we have more robust problem. Wearable electronic based an automatic voltage regulator can automatically preservesthe terminal voltage of generator at a fixed value under varyingly load and operating temperature. AVR controls output by sensing the output voltage at a power-generating coil and compares it to a stable reference. The combination of fractional order controller with an automatic voltage regulator is proved to be better than conventional controllers.

Key words: AVR, FOC, margins, PSS, IOPID

\section{Introduction}

Oustaloup is the pioneer in the field of fractional order calculus. It was also known as command robusted order non-entier (CRONE) controller. It is employed in contrastive feedback control systems. In the recent times podlundy a scientist introduced a generalized fractional PID controller called the fractional order PID controller that offers many advantages and advanced than the classic PID controller that includes $\lambda$ (integrative action) and

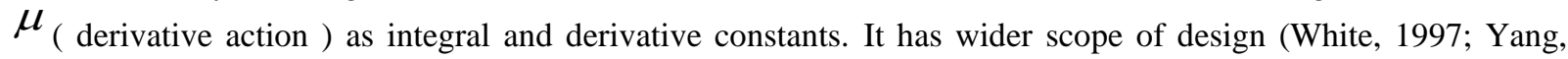
2006; Sampath Kumar, 2020; Samardzic, 2017; Murugan, 2020; Aroulanandam, 2019) this fractional order PID controller is a better solution for many industrial problems and some applications. Generalized FOPID IS

$$
\mathrm{C}(\mathrm{s})=K_{P}+\frac{K_{i}}{S^{\lambda}}+K_{d} S^{\mu}
$$

The foremost work or intention of this FOPID controller is to find out those two constants and fulfill the additional specifications of the systems. Generally FOPID is represented as $P I^{\lambda} D^{\mu}$ when $\lambda=1, \mu=1$, one can get classical PID.

This FOPID increases the system total performance.

\section{Design Specifications}

The aim to design the controller is to achieve the various stipulation regarding sturdiness to plant load disorders, reservations, high frequency noise and frequency domain specifications. In general for any control system gain margin and phase margin are the signs of strength. Further phase of the system is mutually connected to the damping ratio, so it can also be treated as the performance measure (Subhransu, 2011; Duarte, 2015). This system rejects the output disturbances. Steady state can be terminated.

\section{Comparison of PID and FOPID Controllers}

The mathematical model of the conventional PID controller is.

$$
K_{P}+\frac{K_{i}}{S}+K_{d} S
$$




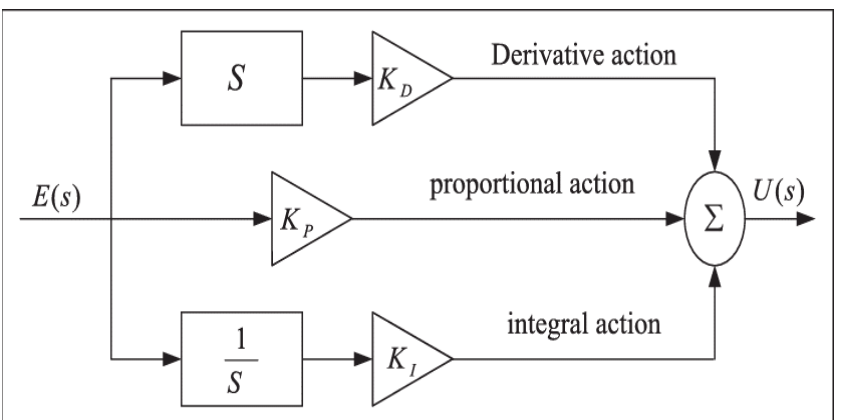

Figure 1. Block diagram of PID controller.

Here to find three unknown parameters. So, it is required to design better controller than the classic PID controller in order to avoid disadvantages. So, here fractional order came into existence. The transfer function obtained for this non-conventional order PID controller is as equation (1).

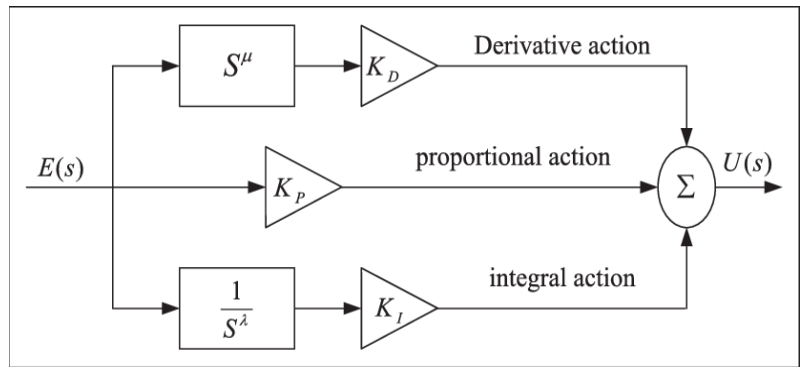

Figure 2. Block diagram of FOPID controller.

The proposed controller satisfies time and frequency parameters, sensitivity function of robustness, load disturbance and high frequency. Further the above specified design specifications influence the stability, stoutness and presentation of the system. So, in order to design this fractional order control system we use an optimization technique, to find all the unknown parameters accurately and improve the features of stability and performance (Grizzle, 2003). For this comparison, further we also design classic PID controller and tested on a physical real system (AVR, PSS) (Hoque, 2014; Igor, 2001; Latchoumi, 2017; Manue, 2006) and prove that fractional order controller gives the better results than the traditional PID controller because these dynamic module characteristics of the fractional PID controller (real dynamic system) is superior than the typical PID controller. Now the conditions regarding phase margin, gain margin, sensitivity functions and sturdiness limitations will be done in the process, further the effective design features regarding presentation, steadiness and sturdiness, of course other varieties of features can also satisfied, related to individual necessities of the structure. We have design features as follows: Gain and Phase margin always work for the significant inventions of strength (i.e., these are the main specifications to design strength of a system). The gain and phase margin specifications are specified by the following equations

$$
\begin{gathered}
|C(j \omega) G(j \omega)|_{d B}=0 d B \\
\arg (C(j \omega) G(j \omega))=\pi+\phi_{m}
\end{gathered}
$$

The third design speciation is the Tuning problem that forces the phase of the system $F(s)=C(s) G(s)$ to be get at where gain is $0 \mathrm{~dB}$ i.e., more or less unvarying at an interval roughly gain $0 \mathrm{~dB}$ frequency region.

$$
\frac{\operatorname{darg}(\mathrm{F}(\mathrm{S}))}{\mathrm{d} \omega} \mid \omega=\omega_{\mathrm{cg}}
$$

High frequency noise rejection: An element corresponds to sensitivity function $T(j \omega)$ given by

$$
\begin{gathered}
T(j \omega)=\left|\frac{C(j \omega) G(j \omega)}{1+C(j \omega) G(j \omega)}\right|_{d B} \leq A d B \\
\forall \omega \geq \omega_{t} \mathrm{rad} / \mathrm{sec} \Rightarrow\left|T(j \omega)_{t}\right|_{d B}=A d B
\end{gathered}
$$

To ensure a output disturbance rejection: A element on the sensitivity function $S(j \omega)$ is given by 


$$
\begin{aligned}
& \left|S(j \omega)=\frac{1}{1+C(j \omega) G(j \omega)}\right|_{d B} \leq B d B \\
& \forall \omega=\omega_{s} \mathrm{rad} / \mathrm{sec} \Rightarrow|S(j \omega)|_{d B}=B d B
\end{aligned}
$$

\section{Designing of FOPID Controller}

In order to know the finest blueprint specifications of Fractional order PID controller. We need to design both fractional order and general PID controllers. We need to design the FOPID controller for the systems AVR,PSS. The transfer function for the FOPID is

$$
C(S)=K_{p}+\frac{K_{i}}{S^{\lambda}}+K_{d} S^{\mu}
$$

To devise this fractional order PID controller, the primary thing need to do is, search for the worst case frequency from the procedure. Initially first we assign the initial specification i.e. the gain cross over frequency and the desired phase margin. The desired phase margin is 40 degrees, from the transfer function the undamped frequency is almost close to $18 \mathrm{krad} / \mathrm{sec}$ and the left half plane zero is about $83 \mathrm{krad} / \mathrm{sec}$. The switching frequency of the converter is found to be $200 \mathrm{kHz}$ (approx $1250 \mathrm{krad} / \mathrm{sec}$ ). So, gain cross over frequency should be almost $1 / 5$ of this value so that might be $250 \mathrm{krad} / \mathrm{sec}$.

Now, with these initial specifications, we start to search for the worst frequency case and it might be $40 \mathrm{krad} / \mathrm{sec}$. For designing the FOPID, We need the specifications initially.

\subsection{Phase Margin Constraint}

$$
\arg \left(G\left(j \omega_{c}\right)\right)=\arg \left(C\left(j \omega_{c}\right) P\left(j \omega_{c}\right)\right)=\pi+\phi_{m}
$$

\subsection{Gain Cross Over Frequency Constraint}

$$
\left|G\left(j \omega_{c}\right)\right|_{d B}=\left|C\left(j \omega_{c}\right) G\left(j \omega_{c}\right)\right|_{d B}=0
$$

\subsection{Robustness to Loop Gain Variation Constraint}

$$
\left|\frac{d(\arg (G(j \omega)))}{d \omega}\right|_{\omega=\omega_{c}}=0
$$

\section{System under consideration}

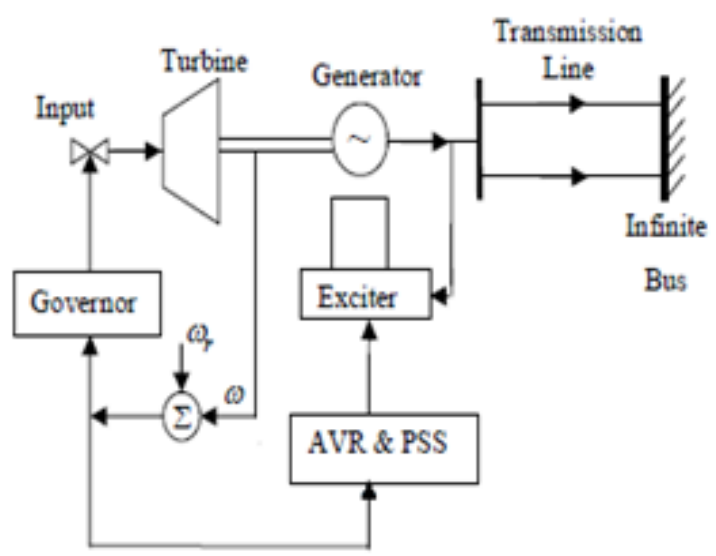

Figure 3. Block diagram of synchronous generator.

From figure 3 below, the flow of energy is explained as, the input coming on to turbine is governed by the $g$ over nor and the turbine is attached to the shaft of the generator the electrical power coming out is passed on to the transmission lines. In this arrangement, the exciter of generator is excited by the part of the power from the generator itself. Many more interconnections are in exciter, but not for discussion in this context. The recital of governor governance is performed by the output of the turbine and the incoming input from the turbine through a proper check between the reference torque $r$. in practice the recital of governor and the exciter are on the surface 
taken care of by the power system stabilizer (PSS) and the automatic voltage regulator (AVR) for invariable input without any break. A PSS installed in the AVR of the Generator improves the power system stability. Automatic Voltage Regulator (AVR)is a device that automatically maintains the output voltage at a set value i.e. it turns the fluctuating voltage level to an unvarying level. This progression is done by varying the load and working temperature. This voltage output at generator is controlling by the AVR is done by sensing the output voltage at a power generating coil and compares it with a reference value.

\section{Operating Theory}

Essentially these automatic voltage regulators are used in alternators in order to bound the voltage to in and around $14.5 \mathrm{~V}$, in order to protect the electrical systems from fatal damage regular failures. AVR's are also used in automobile alternators, transformers, power station generator plants in order to control the output (Manue, 2004; Kanaga, 2015). In general it is negative feedback control system that measures the output voltage and that voltage is compared with desired point the difference of these two signals is generated as error, which is used to adjust the excitation of the generator. As the load and temperature are varied the excitation current in the field winding is varied (increases) as a result the terminal voltage increases. And hence these variations in the voltage are controlled by Automatic Voltage Controller.

\section{Power System Stabilizer (PSS)}

It is device that is used to measure the improvement in the system stability, when it is added to the AVR. It is a device that is provided additionally (Ahmad, 2006). This device is used to damp the electro-mechanical oscillations obtained in the generator in order to protect the shaft line and also to provide stabilization to the network. Only AVR cannot make the fluctuations control and damp, so we add PSS in order to make the process faster (Adams, 2000; Vasanth, 2017). As it is an additional part for Automatic Voltage generator and it is also a device that is able to withstand or recover quickly from the difficult conditions (i.e. like voltage sag or dip along with the faults). When it is given to the AVR, it will ensure the voltage stability by taking the feedback voltage from the network and the excitation (Abhaya , 2015; Hyo-Sung, 2010; Mahmut, 2015; Monje, 2010; Shankar, 2020; Sekaran, 2020). This is mainly used only in the synchronous generators because the speed in those will be depending on the frequency and number of poles and at a certain point speed will be constant. Hence the angles of load that varies can be easily calculated. So we use these more in synchronous generators.

\section{Results}

Now for comparing the performance FOPID and IOPID controllers we have to draw the bode of the open loop plant.

The table below shows the conventional compensation and proposed compensation of FOPID and IOPID Controllers.

Table 1: summary of results

\begin{tabular}{|c|c|c|c|}
\hline $\begin{array}{c}\text { Compensation } \\
\text { types }\end{array}$ & Parameter value & $\begin{array}{c}\text { Peak } \\
\text { overshoot }\end{array}$ & $\begin{array}{c}\text { Settling } \\
\text { time }\end{array}$ \\
\hline FOPID for AVR & $K_{P}=0.01417$ & 1.28 & $11 \mathrm{sec}$ \\
& $k_{i}=0.00317$ & & \\
& $K_{d}=.02155$ & & \\
& $\mu=.50188$, & & \\
& $\lambda=0.43208$ & & \\
& $\mathrm{P} . \mathrm{M}=62 \mathrm{deg}$ & & \\
\hline \multirow{2}{*}{ PID for AVR } & $K_{P}=0.01435$ & 1.5 & \\
& $k_{i}=0.01435$ & & \\
& $K_{d}=0.01966$ & & \\
& & & \\
& & & \\
&
\end{tabular}




\begin{tabular}{|c|c|c|c|}
\hline & $K_{P}=0.97537$ & 1.19 & $13 \mathrm{sec}$ \\
& $k_{i}=0.032878$, & & \\
FOPID for & $K_{d}=.035261$, & & \\
PSS & $\mu=.49968$, & & \\
& $\lambda=0.39485$ & & \\
& $\mathrm{P} . \mathrm{M}=41 \mathrm{deg}$ & & \\
\hline \multirow{2}{*}{ PID for PSS } & $K_{P}=0.00973$ & 1.46 & $73.4 \mathrm{sec}$ \\
& $k_{i}=0.01834$ & & \\
& $K_{d}=0.00298$ & & \\
\hline
\end{tabular}

From the figure below it is shown that both FOPID and IOPID controllers has similar (almost) bandwidth but some difference can be seen. Further proposed technique of FOPID compensation obtains 40 degrees of phase margin across the bandwidth for all frequencies whereas that IOPID controller has approximately 40 degrees phase margin but not the same. From the frequency plot that the conventional compensation based FOPID and IOPID has cut across the stability limit of negative180 degrees line twice, so for the stability to be analyzed properly step and bode plots has to be considered.

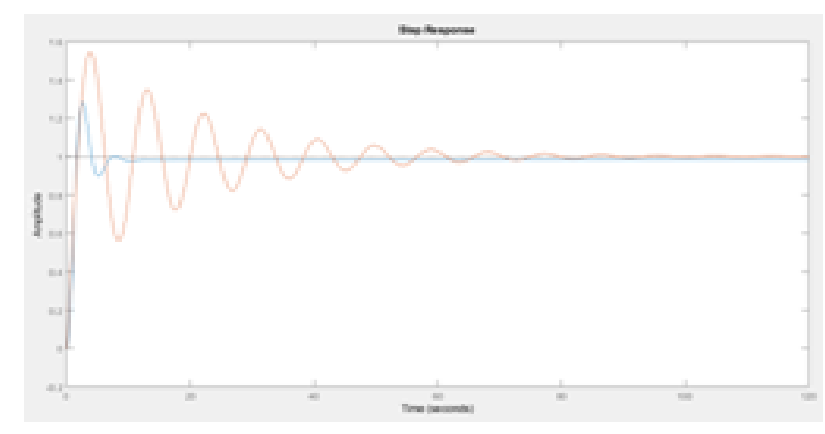

Figure 4 Step response of AVR

Figure 4 above shows the step response of the systems. The settling times are $68.9 \mathrm{sec}$ and $11 \mathrm{sec}$ for IOPID and FOPID respectively. The peak over shoots is 1.55 and 1.21 for IOPID and FOPID respectively. So, finally FOPID gives the better responses than IOPID. Blue represents the FOPID step response and orange represents the IOPID step response.

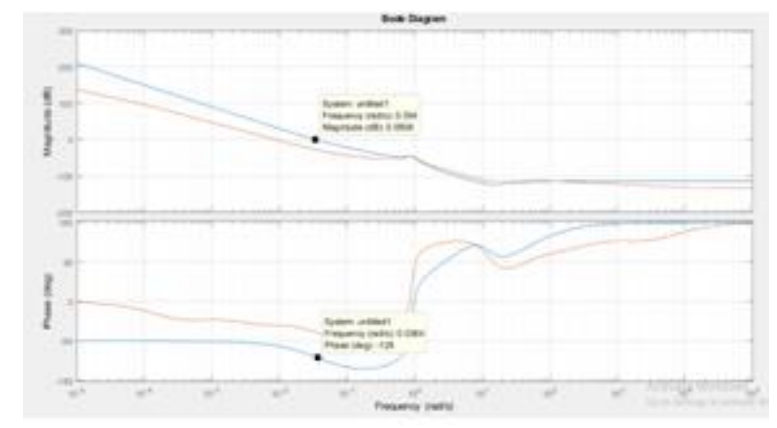

Figure 5 Bode response of AVR

Even the bode plot also helpful in finding the stability of the system. The results are given above. The bode plot of the Fractional order PID gives the enhanced result than the integer order PID controller. Here the FOPID gives 62 degrees of phase margin which is very stable. 


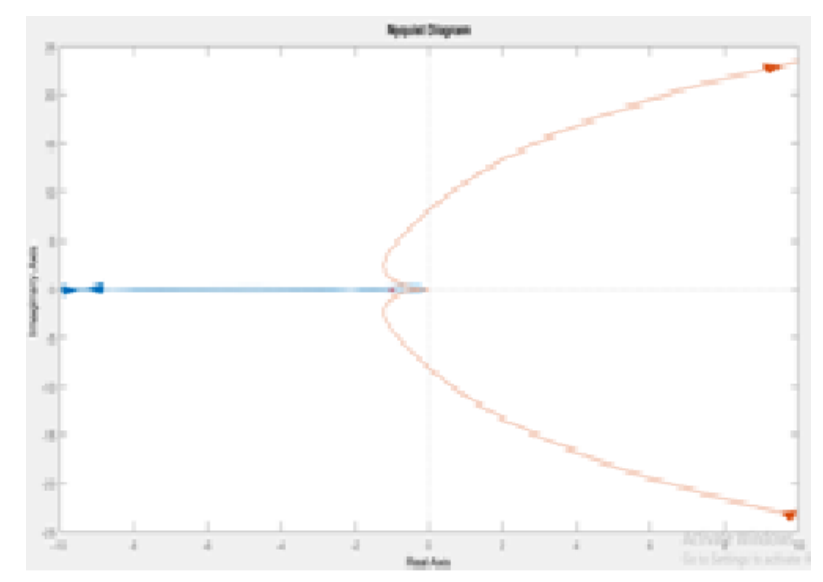

Figure.6 Nyquist response of AVR

Nyquist plot gives the robust stability of the system. When it is left side it is more stable.

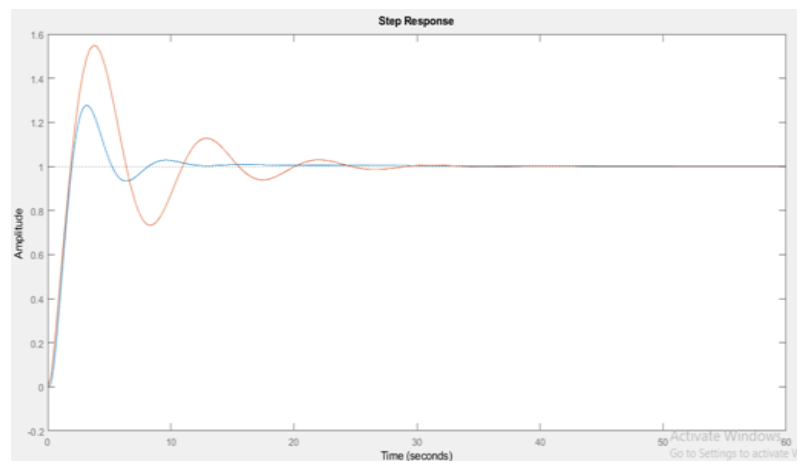

Figure7 Step response of PSS

Figure 7 above shows the step response of the systems. The settling times are $73.4 \mathrm{sec}$ and $13 \mathrm{sec}$ for IOPID and FOPID respectively. The peaks over shoots are 1.46 and 1.19 for IOPID and IOPID respectively. So, finally FOPID gives the better responses than IOPID. Blue represents the FOPID step response and orange represents the IOPID step response.

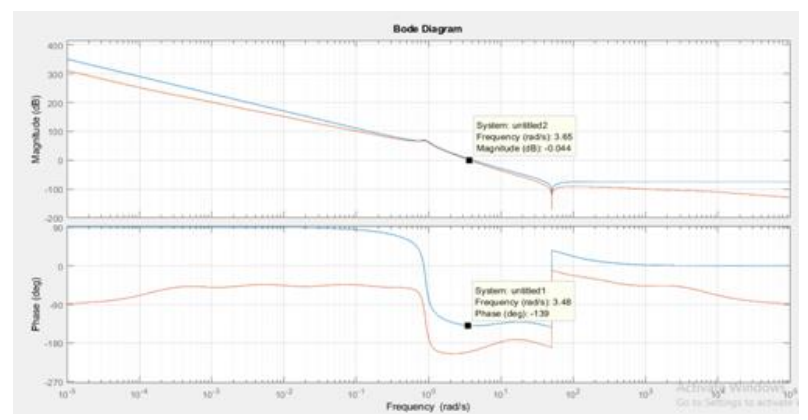

Figure 8 Bode plot of PSS

Even the bode plot also helpful in finding the stability of the system. The results are given above. The bode plot of the FOPID gives the better result than the IOPID controller. Here the FOPID gives 41 degrees of phase margin which is stable. Nyquist plot gives the robust stability of the system. 


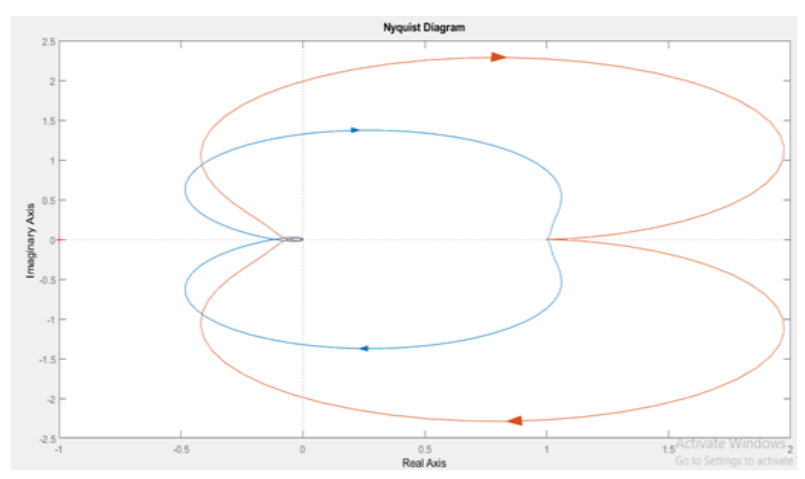

\section{Conclusion}

Figure 9 Nyquist plot of PSS

Finally, in the script, a better method is employed for designing the FOPID controller for decreasing system. This technique provides the improvements those can't be achieved by the classic method. This design process is in the frequency domain by means of the gain cross over frequency and phase margin, with the method or the technique we used, we got the better results and better closed loop performance and the best Robustness properties. The peak overshoot of the system is considered small and stable property comes more when compare to the classic method. The bandwidth found same for both the methods but FOPID found more stable and Robust. From the simulation finally we can conclude that the results in the FOPID controller outperform the IOPID controller in the terms of fulfilling the design specifications i.e., phase margin, gain margin, and robustness. The results finally provide the fact that fractional controller outperforms integer controller for same set of timing constraints.

\section{References}

1. Abhaya Pal Singh, Himanshu Agarwal, Pallavi Srivastava, (2015) "Fractional Order Controller Design For Inverted Pendulum On A Cart System (POAC)" WSEAS TRANSACTIONS on SYSTEMS and CONTROL e-ISSN: 2224-2856. 172. 10,

2. Adams J. .L, T. T. Hartley and C. F. Lorenzo. Fractional-order system identification using complex order-distributions, 200-205p.

3. Ahmad Nor Kasruddin Bin Nasir ( 2006/2007) "Modelling and Controller design for an Inverted Pendulum System”, “Configuration of Automatic Voltage Regulator”, Samsung, Venus, Microtech, Venstab and Silicon, http://www.vener7.com/servo-voltage-stabilizer/.

4. Aroulanandam, V. V., Latchoumi, T. P., Bhavya, B., \& Sultana, S. S. (2019). Object Detection in Convolution Neural Networks Using Iterative Refinements. Revue d'Intelligence Artificielle, 33(5) 367372

5. Grizzle, Moog, And Chevallereau (2003). Nonlinear Control Of Mechanical Systems With An Unactuated Cyclic Variable, Submitted To IeeeTac.

6. Hoque M. .M, (2014). "Design, implementation and performance study of programmable automatic voltage regulator”, Electrical Systems 10-4, pp.472-483.

7. Hyo-Sung Ahn, VarshaBhambhani and YangQuan Chen. Fractional-order integral and derivative controller design for temperature profile control, 4766-4771p.

8. Igor Podlubny. Fractional Differential Equations. 198-1st Edition, Elsevier.

9. KanagaSubaRaja S., S. Usha Kiruthika, (2015) 'An Energy Efficient Method for Secure and Reliable Data Transmission in Wireless Body Area Networks Using RelAODV', International Journal of Wireless Personal Communications, ISSN 0929-6212, 83(4), pp. 2975-2997. https://doi.org/10.1007/s11277-0152577-x (IF: 1.061)

10. Latchoumi, T. P., Loganathan, J., Parthiban, L., \& Janakiraman, S. (2016, August). OFS method for selecting active features using clustering techniques. In Proceedings of the International Conference on Informatics and Analytics (pp. 1-4).

11. Latchoumi, T. P., \& Parthiban, L. (2016). Secure Data Storage in Cloud Environment using MAS. Indian Journal of Science and Technology, 9, 24-29.

12. Latchoumi, T. P., \& Kannan, V. V. (2013). Synthetic Identity of Crime Detection. International Journal, 3(7), 124-129.

13. Latchoumi, T. P., Kannan, V. V., \& Ezhilarasi, T. P. (2013). Leasing Processing Power from Mid network using Wireless Communication. International Journal, 3(5), 191-199. 
14. MahmutReyhanoglu,Arjan van der Schaft,N. Harris McClamroch and IlyaKolmanovsky. (2015). Dynamics and Control of a Class of Under actuated Mechanical Systems, WSEAS TRANSACTIONS on SYSTEMS and CONTROL. E-ISSN: 2224-2856. 172(10),

15. Manue F. Silva, J. A. Tenreiro Machado. (2006). Fractional Order PD_Joint Control of Legged Robots, Journal of Vibration and Control, 12(12): 1483-1501.

16. Manue F. Silva, J. A. Tenreiro Machado and A. M. Lopes. (2004). Fractional Order Control of a Hexapod Robot, Nonlinear Dynamics 38 (1-4), 417-433

17. Murugan, S., Jeyalaksshmi, S., Mahalakshmi, B., Suseendran, G., Jabeen, T. N., \& Manikandan, R. (2020). Comparison of ACO and PSO algorithm using energy consumption and load balancing in emerging MANET and VANET infrastructure. Journal of Critical Reviews, 7(9).

18. Samardzic, M.P.Lazarevic, B. Cvetkovic. Optimal conventional and fractional PID control algorithm for a robotic system with three degrees of freedom driven by DC motors, 297-301.

19. Sampathkumar, A., Murugan, S., Sivaram, M., Sharma, V., Venkatachalam, K., \& Kalimuthu, M. (2020). Advanced Energy Management System for Smart City Application Using the IoT. In Internet of Things in Smart Technologies for Sustainable Urban Development (pp. 185-194). Springer, Cham.

20. Sekaran, K., Rajakumar, R., Dinesh, K., Rajkumar, Y., Latchoumi, T. P., Kadry, S., \& Lim, S. (2020). An energy-efficient cluster head selection in wireless sensor network using grey wolf optimization algorithm. TELKOMNIKA, 18(6), 2822-2833.

21. Shankar, G., Latchoumi, T. P., Chithambarathanu, M., Balayesu, N., \& Shanmugapriya, C. (2020). An Efficient Survey on Energy Conservation System with Video Surveillance. Journal of Xian University of Architecture and Technology, 12(7), 100-106.

22. SubhransuPadhee, AbhinavGautam, Yaduvir Singh, and Gagandeep Kaur. (2011) A Novel Evolutionary Tuning Method for Fractional Order PID Controller, (IJSCE) ISSN: 2231-2307, 1(3).

23. Duarte Valrio, Jos S da Costa. Non-Integer order control of a flexible robot, 1-6p. "Specifications and Ratings of Practical AVR", http://www.voltagestabilizer.com and http://www.voltageregulator.com.

24. Vasanth, A. V., Venkatachalapathy, K., Latchoumi, T. P., Parthiban, L., Sowmia, T., \& OhmPrakash, V. (2018). An Efficient Cache Refreshing Policy to Improve QoS in MANET Through RAMP. In Proceedings of the Second International Conference on Computational Intelligence and Informatics (pp. 369-381). Springer, Singapore.

25. White J. .R, UMass-Lowell . ( 1997). Lecture Notes "Introduction to the Design and Simulation of Controlled Systems" 24.509,

26. YangQuan Chen, (2006), Ubiquitous Fractional Order Controls, Second IFAC Workshop on Fractional Differentiation and its Applications, 2(1), 481-492p. 